\title{
Expression and Clinical Value Analysis of CSF2RA in Malignant Tumor Based on Database
}

\section{Fengling Chen}

National Center for International Research of Bio-Targeting Theranostics, Guangxi Key Laboratory of Bio-Targeting Theranostics, Collaborative Innovation Center for Targeting Tumor Diagnosis and Therapy, Guangxi Talent Highland of Bio-Targeting Theranostics, Guangxi Medical University, Nanning, China

Email: chenfengling@stu.gxmu.edu.cn

How to cite this paper: Chen, F.L. (2021) Expression and Clinical Value Analysis of CSF2RA in Malignant Tumor Based on Database. Journal of Biosciences and Medicines, 9, 149-157.

https://doi.org/10.4236/jbm.2021.94013

Received: March 21, 2021

Accepted: April 22, 2021

Published: April 25, 2021

Copyright $\odot 2021$ by author(s) and Scientific Research Publishing Inc. This work is licensed under the Creative Commons Attribution International License (CC BY 4.0).

http://creativecommons.org/licenses/by/4.0/

\begin{abstract}
Objective: to explore the expression and clinical value of CSF2RA in pan-cancer. Methods: the data was extracted from ONCOMINE, Human Protein Atlas and Kaplan Meier-plotter. The expression level of CSF2RA on cancer tissues and normal tissues, the relationship between CSF2RA and overall survival of cancer patients were analyzed, respectively. Results: CSF2RA mRNA was over-expressed on breast cancer, colorectal cancer, kidney cancer and liver cancer, and CSF2RA protein was over-expressed on melanoma. The expression level of CSF2RA was not associated with overall survival of cancer patients significantly. Conclusion: CSF2RA is over-expression on certain cancers and most immune cells, which maybe contributes to activation of immune system. The high expression of CSF2RA protein on melanoma is maybe associated with adverse outcome of application of GM-CSF.
\end{abstract}

\section{Keywords}

CSF2RA, GM-CSF, Cancer, Immune Cells

\section{Introduction}

CSF2RA, "Colony stimulating factor 2 receptor alpha subunit (CD116)", is a receptor for GM-CSF (granulocyte-macrophage colony-stimulating factor), and plays a vital role for proliferation, differentiation and functional activation of hematopoietic cells such as macrophages and leukocytes [1]. The absence of GM-CSF or disruption of CSF2RA/GM-CSF pathway would interfere the differentiation of macrophages. It's been reported that CSF2RA is at over-expression in cancer tissues compared to normal tissues, and could enhance immune activity in tumor microenvironment [1] [2]; however, the internal mechanism about it hasn't been explored clearly. Similarly, the ligand, GM-CSF, has been ap- 
proved for clinical treatment of malignant tumor as an immune adjuvant, to initiate more robust activation of $\mathrm{CD}^{+}$and $\mathrm{CD}^{+} \mathrm{T}$ cells, induce rapid maturation of granulocytes, macrophages and specific antigen presentation reaction of dendritic cells and improve neutropenia subsequent after chemotherapy and radiotherapy. Nevertheless, it was reported that the GM-CSF could promote angiogenesis around tumors, inhibit immune response for tumor and facilitate to tumor relapse when GM-CSF was used for anti-tumor therapy, and the GM-CSF seduced negative cytokines to modulate immune suppression, which was possibly related with the expression of CSF2RA protein in tumor tissue [3] [4] [5]. Hence, this study supposes that CSF2RA is associated with cancer significantly, and analyzes expression difference between cancer and normal tissues, relationship between CSF2RA and overall survival of cancer patients, explores its clinical value by mining ONCOMINE, HPA and Kaplan Meier-plotter databases.

\section{Methods}

\subsection{Data Retrieval and Preprocessing from ONCOMINE Data}

The ONCOMINE data (https://www.oncomine.org/) and the screening conditions are as follows: "Gene: CSF2RA"; "Analysis Type: Cancers vs. Normal Analysis"; "Data Type: DNA and mRNA".

\subsection{Data Retrieval and Preprocessing from Human Protein Atlas Data}

The HPA data (https://www.proteinatlas.org/) and the screening conditions are as follows: "Gene: CSF2RA"; the data were collected from "tissue", "cell" and "pathology" columns, respectively.

\subsection{Data Retrieval and Preprocessing from Kaplan Meier-Plotter}

The Kaplan Meier-plotter (http://kmplot.com/analysis/) and the screening conditions are as follows: "Gene Type: mRNA"; "Cancer Type: pan-cancer"; "Gene symbol: CSF2RA"; "Split patients by: Auto select best cutoff”; "Survival: OS”.

\section{Results}

\subsection{Expression of mRNA of CSF2RA in Multiple Cancers}

There were 378 researches about expression of mRNA of CSF2RA in cancer compared to normal tissues collected and derived from ONCOMINE data, and 219 researches presented the difference of expression was significant. Among them, 116 researches had a conclusion that CSF2RA is over-expression in malignant tumor compared to normal tissue; on the contrary, 103 researches presented that CSF2RA is under-expression in malignant tumor compared to normal tissue (Figure 1). In addition, the result from HPA data showed that mRNA of CSF2RA was expressed in 17 kinds of cancer tissues and the expression in glioma was at the most high level (Figure 2). Next, the meta-analysis was performed for these researches, and the results were shown in Table 1. The 


\begin{tabular}{|c|c|}
\hline $\begin{array}{l}\text { Analysis Type } \\
\text { by Cancer }\end{array}$ & $\begin{array}{l}\text { Cancer } \\
\text { vs. } \\
\text { Normal }\end{array}$ \\
\hline Bladder Cancer & 1 \\
\hline Brain and CNS Cancer & 10 \\
\hline Breast Cancer & 12 \\
\hline Cervical Cancer & 2 \\
\hline Colorectal Cancer & 10 \\
\hline Esophageal Cancer & 1 \\
\hline Gastric Cancer & 5 \\
\hline Head and Neck Cancer & 12 \\
\hline Kidney Cancer & 14 \\
\hline Leukemia & 11 \\
\hline Liver Cancer & 4 \\
\hline Lung Cancer & 2 \\
\hline Lymphoma & 19 \\
\hline Melanoma & 1 \\
\hline Myeloma & 4 \\
\hline Other Cancer & 7 \\
\hline Ovarian Cancer & 2 \\
\hline Pancreatic Cancer & 2 \\
\hline Prostate Cancer & 4 \\
\hline Sarcoma & 4 \\
\hline Significant Unique Analyses & $116 \quad 103$ \\
\hline Total Unique Analyses & 378 \\
\hline
\end{tabular}

Figure 1. Difference of expression of CSF2RA in cancers.

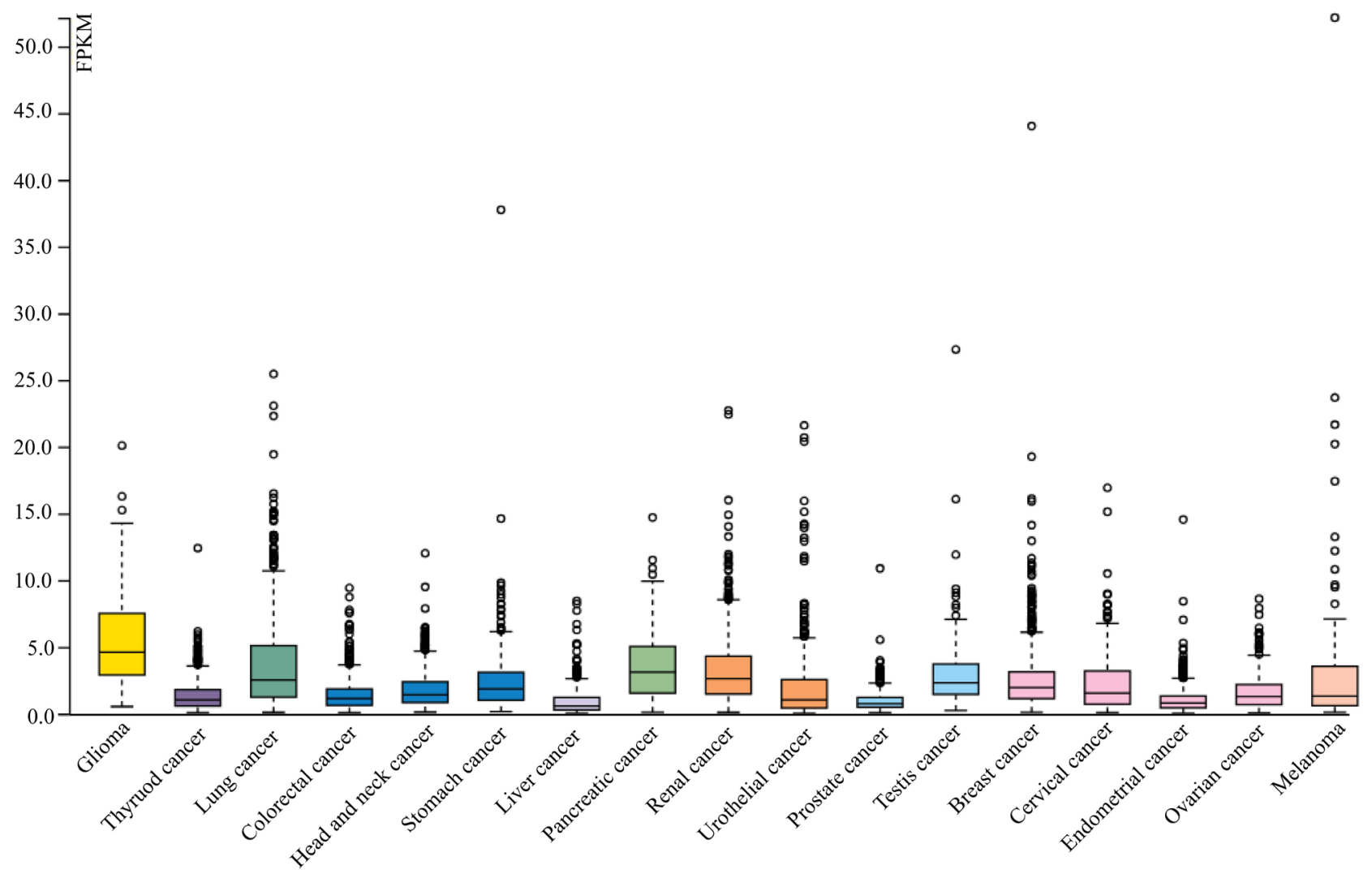

Figure 2. Expression level of CSF2RA in cancers. 
Table 1. The meta-analysis about expression level of CSF2RA in cancers.

\begin{tabular}{|c|c|c|c|c|}
\hline Cancer Type & Over/Under Expression & Median Rank & p-Value & Numbers of Analyses \\
\hline Bladder Cancer & Over & $11,110.0$ & 0.896 & 9 \\
\hline Brain and CNS Cancer & Over & 3324.0 & 0.248 & 24 \\
\hline Breast Cancer & Over & 7157.0 & 0.009 & 29 \\
\hline Cervical Cancer & Over & 6925.5 & 0.209 & 6 \\
\hline Colorectal Cancer & Over & 7182.0 & 0.024 & 24 \\
\hline Esophageal Cancer & Over & 7117.0 & 0.608 & 9 \\
\hline Gastric Cancer & Over & 8260.0 & 0.191 & 25 \\
\hline Head and Neck Cancer & Over & 4078.5 & 0.477 & 26 \\
\hline Kidney Cancer & Over & 1253.5 & $9.40 \mathrm{E}-5$ & 16 \\
\hline Leukemia & Over & 3250.0 & 0.416 & 16 \\
\hline Liver Cancer & Over & 728.5 & 0.002 & 6 \\
\hline Lung Cancer & Over & 7927.0 & 0.511 & 17 \\
\hline Lymphoma & Over & 1766.5 & 0.300 & 28 \\
\hline Melanoma & Over & 6635.0 & 0.289 & 5 \\
\hline Myeloma & Over & 5634.0 & 0.006 & 7 \\
\hline Ovarian Cancer & Over & 5809.0 & 0.232 & 13 \\
\hline Pancreatic Cancer & Over & 4077.0 & 0.139 & 9 \\
\hline Prostate Cancer & Over & 5022.0 & 0.042 & 13 \\
\hline Sarcoma & Over & $10,023.0$ & 0.275 & 17 \\
\hline
\end{tabular}

over-expression of mRNA of CSF2RA in breast cancer $(p=0.009)$, colorectal cancer $(p=0.024)$, kidney cancer $(p=9.40 \mathrm{E}-5)$, liver cancer $(\mathrm{p}=0.002)$, myeloma $(\mathrm{p}=0.006)$ and prostate cancer $(\mathrm{p}=0.042)$ tissues compared to normal tissues were significant.

\subsection{Expression of Protein of CSF2RA in Multiple Cancers}

The expression levels of CSF2RA protein in 20 kind of malignant tumors were summarized from HPA data, as shown in Figure 3. In most cancer tissues of patients, the expression of protein was negative, however, the positive rate of protein in melanoma could reach at $42 \%$. Moreover, the expression level was moderate and even high in melanoma tissues and the protein was primarily expressed on cytoplasm of tumor cells.

\subsection{CSF2RA and the Prognosis of Cancer Patients}

The overall survival analysis of breast cancer, colorectal cancer, kidney cancer and liver cancer patients with over-expression of CSF2RA were accomplished by Kaplan Meier-Plotter data. The results showed there was no significant difference about the overall survival between patients at low or high expression levels of CSF2RA with breast cancer ( $P>0.05)$, colorectal cancer $(P>0.05)$, kidney 


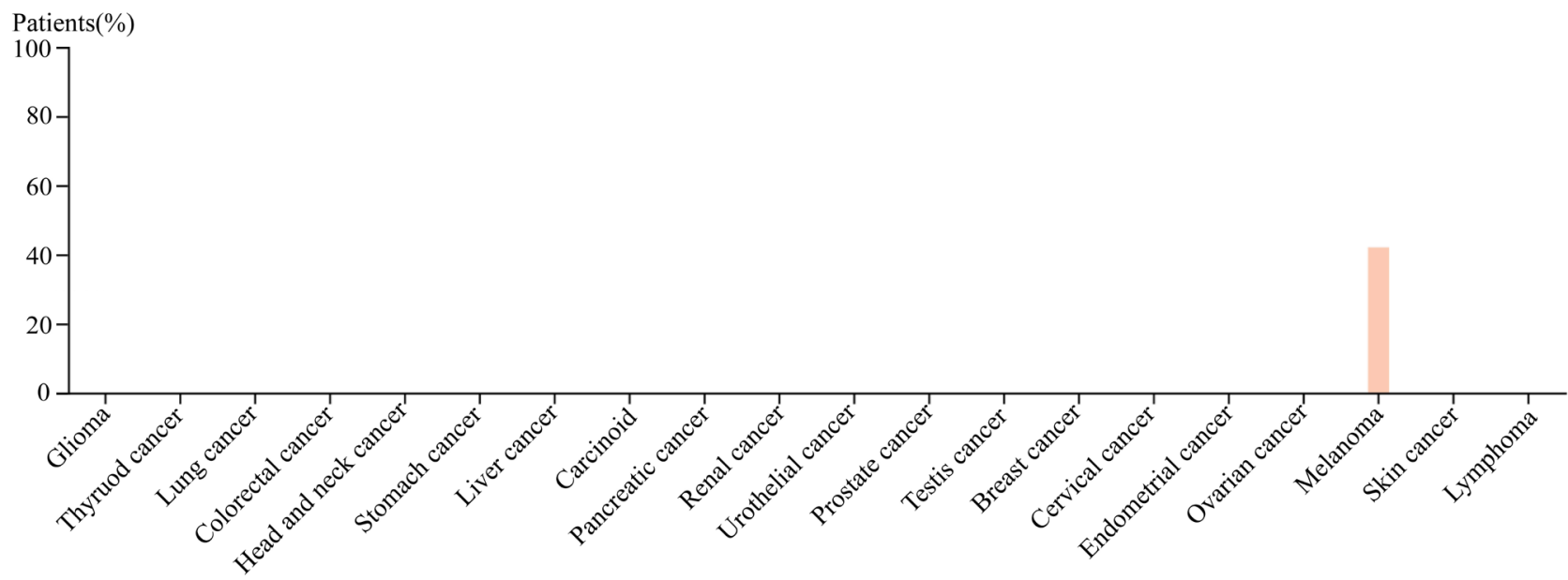

Figure 3. Expression of protein of CSF2RA in multiple cancers.

cancer $(\mathrm{P}>0.05)$ and liver cancer $(\mathrm{P}>0.05)$, respectively. Therefore, it's considered that the expression levels of CSF2RA were not associated with overall survival of cancer patients and CSF2RA couldn't be a prognostic factor of cancer patients (Figure 4).

\subsection{Expression of CSF2RA in Human Tissues and Cells}

The results from HPA data indicated that the expression of CSF2RA is at high level in Granulocytes, Dendritic cells and Monocytes in immune system, and Placenta tissue (Figure 5(A)). The expression level of CSF2RA on granulocytes was higher than other cells. Beside of placenia tissue, the expressions of CSF2RA on other tissues were relatively low. Similarly, the expression results of cells showed that expression of CSF2RA on Monocytes is at the most high level (Figure 5(B)). In the blood system, the expression levels of CSF2RA on monocytes, macrophage, kupffer cells and hofbauer cells were relatively higher than other cells.

\section{Discussions}

CSF2RA as a tumor-related gene has been approved to be a drug target by FDA. In this study, mRNA of CSF2RA is over-expression in certain cancers such as breast cancer, colorectal cancer, kidney cancer and liver cancer. And the expression on other cancer patients is not significant. In terms of protein expression, it's significantly positive on melanoma tissue and signal of other cancer tissue was negative, which indicates that the CSF2RA protein may be expressed on melanoma cells specifically. CSF2RA participates the biological process by incorporating GM-CSF ligand. According to Figure 5, the CSF2RA was over-expressed on immune cells, which is related with immune activation function of GM-CSF. The integration of GM-CSF and CSF2RA promotes the maturation of granulocytes, dendritic cells and $\mathrm{T}$ cells, polarization of macrophages and induces ADCC [3] [4] [6] [7]. Moreover, it could trigger strong anti-tumor immune response in cancer treatment. And the GM-CSF/CSF2RA could correct low level of granulocytes to recover immunity of patients undergoing 
A

Breast Cancer $(\mathrm{n}=1089)$

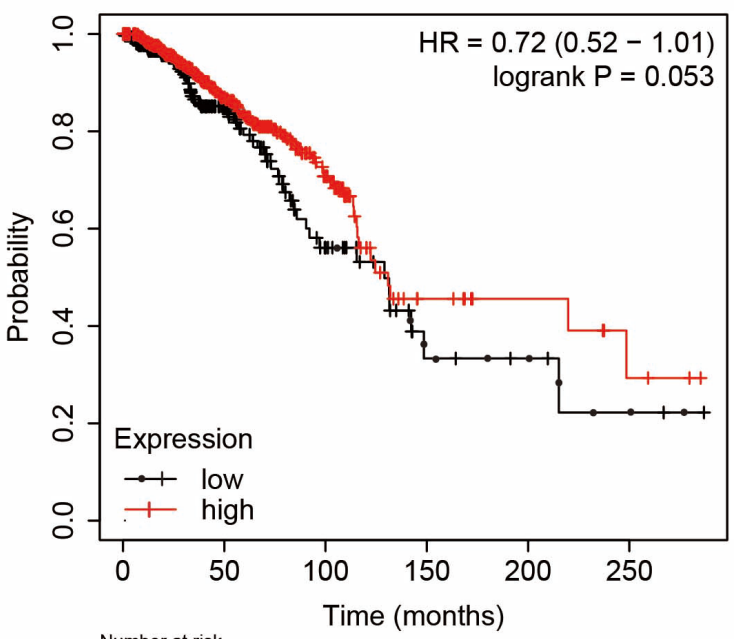

\begin{tabular}{rcccccc}
\multicolumn{7}{c}{ Number at risk } \\
low & 334 & 84 & 26 & 6 & 4 & 2 \\
high & 755 & 236 & 69 & 12 & 7 & 3
\end{tabular}

C

Kidney Cancer $(n=530)$

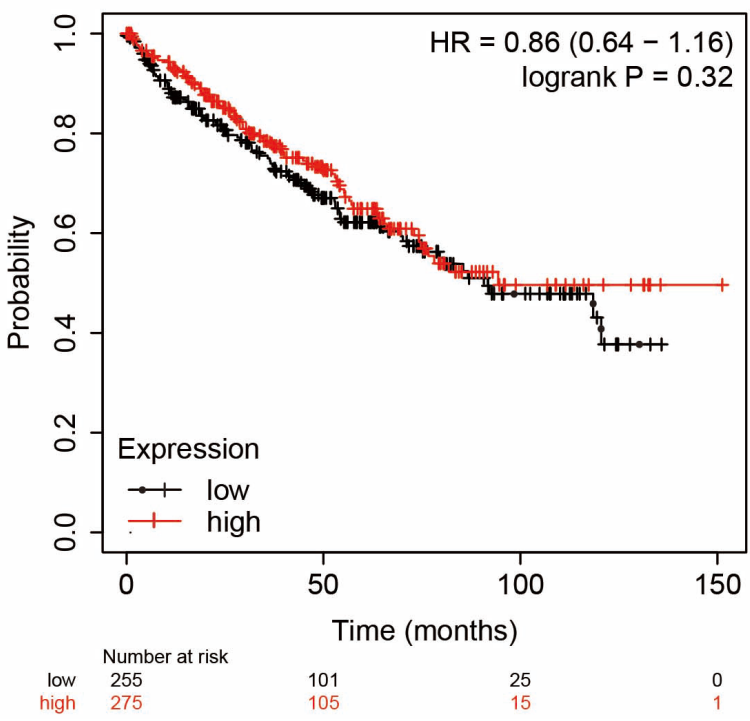

B

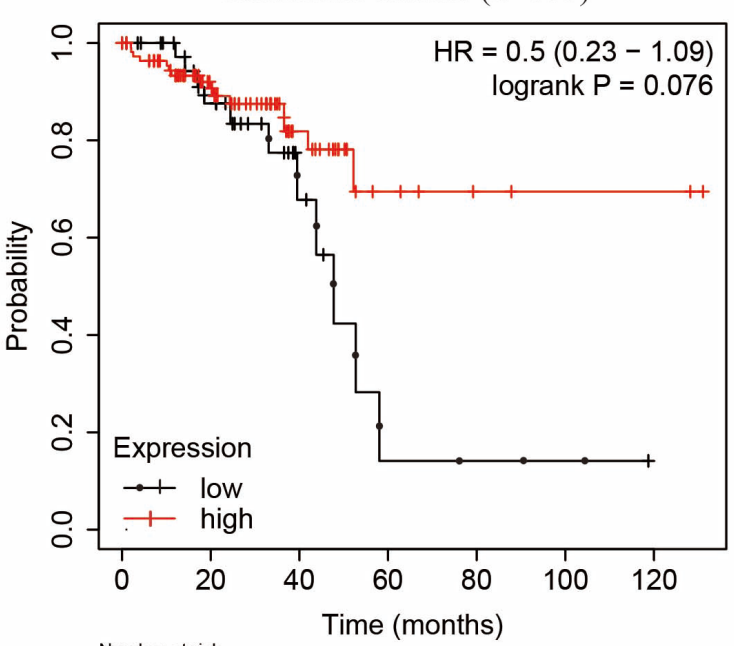

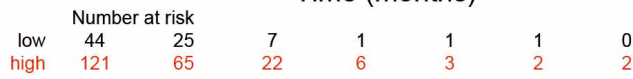

D

Liver Cancer $(\mathrm{n}=370)$

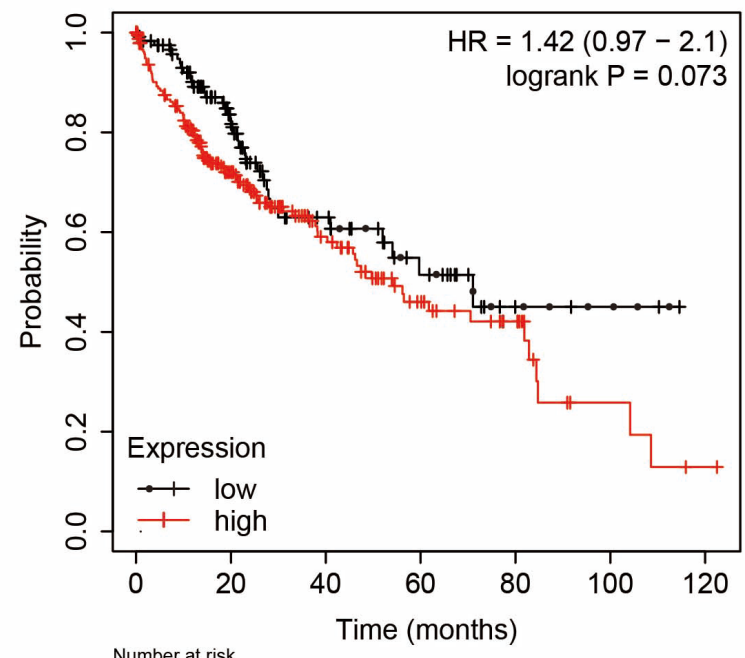

\begin{tabular}{rrrrrrrr}
\multicolumn{7}{c}{ Number at risk } & \\
low & 122 & 65 & 29 & 15 & 3 & 2 & 0 \\
high & 248 & 117 & 55 & 27 & 16 & 4 & 1
\end{tabular}

Figure 4. The survival curves of cancer patients with different levels of CSF2RA expression. (A) Breast Cancer; (B) Colorectal Cancer; (C) Kidney Cancer; (D) Liver Cancer.

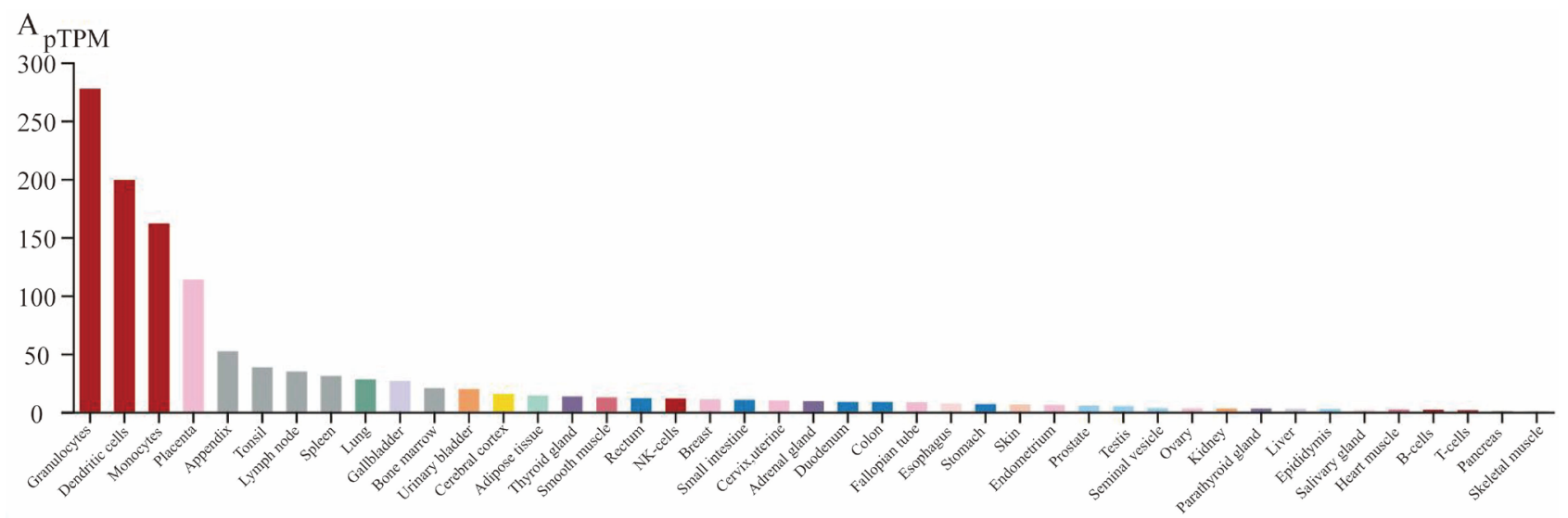




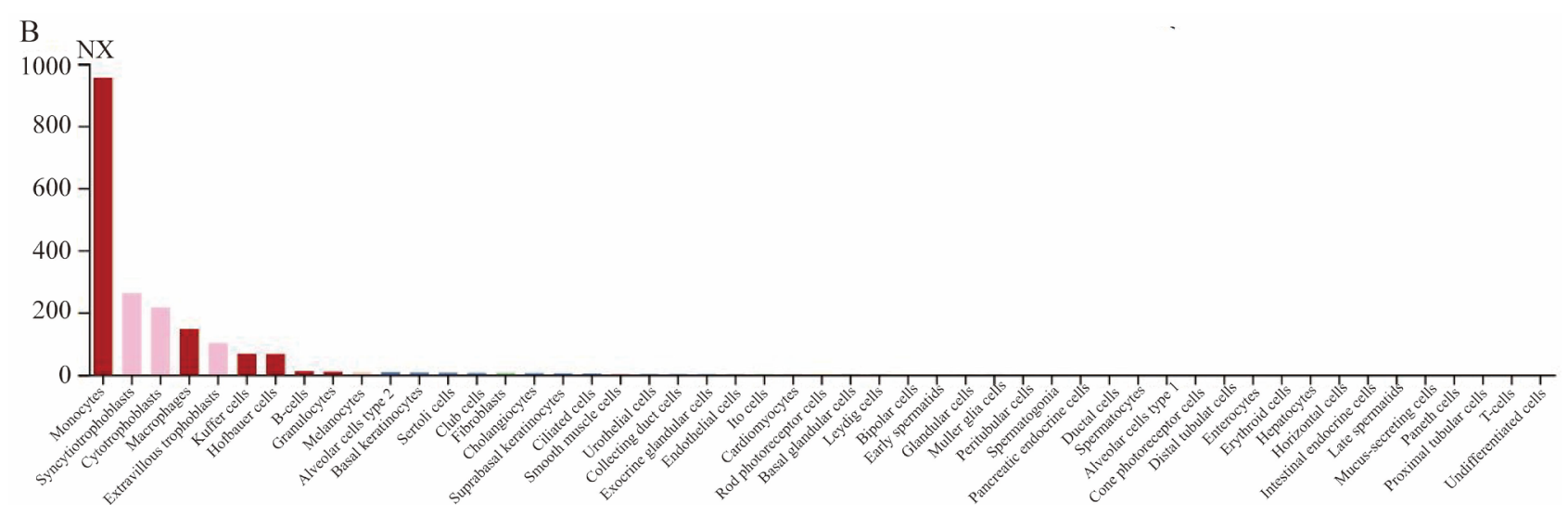

Figure 5. Expression of CSF2RA in human tissues and cells.

chemotherapy and radiotherapy [5] [8]. Although the expression of CSF2RA on cancer tissue is relatively weak, the expression on immune cells such as monocytes and granulocytes is at high level. Therefore, GM-CSF integrates with CSF2RA receptor on immune cells to activate immune system to trigger anti-tumor response in cancer patients. In addition, although it's been considered as a potential prognostic factor for tumor patients, the results showed that there was no difference between survival time of cancer patients at low or high CSF2RA mRNA levels respectively from analysis of database, indicating that the CSF2RA plays an insignificant role in tumor of post-treatment patients possibly without application of GM-CSF drug. And the GM-CSF was contributed to tumor relapse probably [3].

Currently, it has been reported that GM-CSF had potential to induce immune suppression for cancer, even stimulate the progress of cancer by inducing tumor angiogenesis [5] [9] [10] [11] which was associated with high expression level of ligand such as CSF2RA possibly [3]. Interestingly, CSF2RA protein is overexpression on melanoma significantly. A study presented that GM-CSF could recruit tumor-related macrophages into melanoma site and even enhance the proliferation and invasion of melanoma cells [12]. In addition, Slingluffs and $\mathrm{Fa}$ ries reported that the application of GM-CSF immune adjuvant weakens the anti-tumor immune response in vaccine trial on melanoma [13] [14]. Therefore, the over-expression of CSF2RA protein maybe leads to adverse outcome for cancer treatment with the application of GM-CSF. The clinical value and the molecule mechanism for cancer treatment of CSF2RA on cancer cells needs to be further explored.

\section{Acknowledgements}

This work has been supported by Innovation Project of Guangxi Graduate Education (No. YCSW2019108).

\section{Conflicts of Interest}

The author declares no conflicts of interest regarding the publication of this paper. 


\section{References}

[1] Hetzel, M., Suzuki, T., Hashtchin, A.R., et al. (2017) Function and Safety of Lentivirus-Mediated Gene Transfer for CSF2RA-Deficiency. Human Gene Therapy Methods, 28, 318-329. https://doi.org/10.1089/hgtb.2017.092

[2] Jones, T.D., Eble, J.N., Wang, M.S., et al. (2005) Molecular Genetic Evidence for the Independent Origin of Multifocal Papillary Tumors in Patients with Papillary Renal Cell Carcinomas. Clinical Cancer Research, 11, 7226-7233. https://doi.org/10.1158/1078-0432.CCR-04-2597

[3] Aliper, A.M., Frieden-Korovkina, V.P., Buzdin, A., et al. (2014) A Role for G-CSF and GM-CSF in Nonmyeloid Cancers. Cancer Medicine, 3, 737-746. https://doi.org/10.1002/cam4.239

[4] Ragnhammar, P. (1996) Anti-Tumoral Effect of GM-CSF with or without Cytokines and Monoclonal Antibodies in Solid Tumors. Medical Oncology, 13, 167-176. https://doi.org/10.1007/BF02990844

[5] Chu, Y., Wang, L.X., Yang, G., et al. (2006) Efficacy of GM-CSF-Producing Tumor Vaccine after Docetaxel Chemotherapy in Mice Bearing Established Lewis Lung Carcinoma. Journal of Immunotherapy, 29, 367-380. https://doi.org/10.1097/01.cji.0000199198.43587.ba

[6] van de Laar, L., Coffer, P.J. and Woltman, A.M. (2012) Regulation of Dendritic Cell Development by GM-CSF: Molecular Control and Implications for Immune Homeostasis and Therapy. Blood, 119, 3383-3393.

https://doi.org/10.1182/blood-2011-11-370130

[7] Verreck, F.A., de Boer, T., Langenberg, D.M., et al. (2004) Human IL-23-Producing Type 1 Macrophages Promote But IL-10-Producing Type 2 Macrophages Subvert Immunity to (myco)Bacteria. Proceedings of the National Academy of Sciences of the United States of America, 101, 4560-4565.

https://doi.org/10.1073/pnas.0400983101

[8] Urdinguio, R.G., Fernandez, A.F., Moncada-Pazos, A., et al. (2013) ImmuneDependent and Independent Antitumor Activity of GM-CSF Aberrantly Expressed by Mouse and Human Colorectal Tumors. Cancer Research, 73, 395-405. https://doi.org/10.1158/0008-5472.CAN-12-0806

[9] Bronte, V., Chappell, D.B., Apolloni, E., et al. (1999) Unopposed Production of Granulocytemacrophage Colony-Stimulating Factor by Tumors Inhibits CD8+ T Cell Responses by Dysregulating Antigen-Presenting Cell Maturation. The Journal of Immunology, 162, 5728-5737.

[10] Hong, I.-S. (2016) Stimulatory versus Suppressive Effects of GM-CSF on Tumor Progression in Multiple Cancer Types. Experimental \& Molecular Medicine, 48, e242. https://doi.org/10.1038/emm.2016.64

[11] Clive, K.S., Tyler, J.A., Travis Clifton, G., et al. (2010) Use of GM-CSF as an Adjuvant with Cancer Vaccines: Beneficial or Detrimental? Expert Review of Vaccines, 9, 519-525. https://doi.org/10.1586/erv.10.40

[12] Obermueller, E., Vosseler, S., Fusenig, N.E., et al. (2004) Cooperative Autocrine and Paracrine Functions of Granulocyte Colony-Stimulating Factor and Granulocyte-Macrophage Colony-Stimulating Factor in the Progression of Skin Carcinoma Cells. Cancer Research, 64, 7801-7812. https://doi.org/10.1158/0008-5472.CAN-03-3301

[13] Slingluff Jr., C.L., Petroni, G.R., Olson, W.C., et al. (2009) Effect of Granulocyte/Macrophage Colony-Stimulating Factor on Circulating CD8+ and CD4+ T-Cell 
Responses to a Multipeptide Melanoma Vaccine: Outcome of a Multicenter Randomized Trial. Clinical Cancer Research, 15, 7036-7044.

https://doi.org/10.1158/1078-0432.CCR-09-1544

[14] Faries, M.B., Hsueh, E.C., Morton, D.L., et al. (2009) Effect of Granulocyte/Macrophage Colony Stimulating Factor on Vaccination with an Allogenic Whole-Cell Melanoma Vaccine. Clinical Cancer Research, 15, 7029-7035.

https://doi.org/10.1158/1078-0432.CCR-09-1540 